\title{
Clostridium septicum panophthalmitis with systemic complications
}

\author{
MICHAEL S INSLER, ZEYNEL A KARCIOGLU, AND THOMAS NAUGLE JR \\ From the Department of Ophthalmology, Tulane University School of Medicine, 1430 Tulane Avenue, \\ New Orleans, LA 70112, USA
}

SUmmary A fulminant case of endophthalmitis due to Clostridium septicum is described. The patient presented with spontaneous gas gangrene panophthalmitis, with early visual loss and an air bubble in the anterior chamber. Death ensued, and necropsy revealed changes consistent with severe arterosclerotic cardiovascular disease, a relationship not uncommon in patients with clostridium sepsis. This association as well as the histopathology of the globe are discussed.

Gas gangrene panophthalmitis is a rare condition usually following penetrating injury to the globe. In most of the reported cases the infecting organism has been Clostridium perfringens. ${ }^{1-5}$

Characteristic findings are brawny swelling of the lids, coffee coloured discharge, hypopyon, ring abscess of the cornea, gas bubbles in the anterior chamber, and early loss of vision. Systemic manifestations include fever and leucocytosis, malaise, nausea, and vomiting. Treatment generally consists of evisceration or enucleation of the globe and systemic antibiotics. Extraocular extension of infection is prevented by well timed surgical intervention.

To the best of our knowledge, this paper is the first report of gas gangrene panophthalmitis due to Clostridium septicum.

\section{Case report}

A 78-year-old Caucasian woman was admitted to hospital on 15 October 1981 with a 24-hour history of nausea, vomiting, and mild abdominal pain. The diagnosis on admission was dehydration and acute gastroenteritis. There was a history of atherosclerotic heart disease and degenerative arthritis. Vital signs at the time of admission showed a blood pressure of $152 / 80 \mathrm{mmHg}$, respiration rate of $20 / \mathrm{min}$, a slight tachycardia, and a temperature of $101^{\circ} \mathrm{F}\left(38 \cdot 3^{\circ} \mathrm{C}\right)$. Examination revealed a slightly obese Caucasian woman who was vomiting and complaining of abdominal pain. The heart and lung examination was normal. The abdominal examination revealed Correspondence to Michacl S Insler, MD. tenderness but good bowel sounds. The white cell count was $28.9 \times 10^{4} / \mathrm{l}$; the haemoglobin was $13.7 \mathrm{~g} / \mathrm{dl}$, and the packed cell volume was $40 \cdot 1 \%$.

Later on that evening the patient became confused and hypotensive and had a respiratory arrest. She responded to cardiorespiratory resuscitation and was transferred to the intensive care unit. The differential diagnosis at that time included a myocardial infarction and gastrointestinal haemorrhage. A serous discharge was noted from the right eye, and lid and conjunctival specimens were taken for culture. Erythromycin $500 \mathrm{mg}$ intravenously six hourly was added to the cefamandole begun on admission.

Two days after admission while the patient was on a ventilator her temperature spiked to $104.6^{\circ} \mathrm{F}$ $\left(40 \cdot 3^{\circ} \mathrm{C}\right)$ and blood cultures were obtained. A heart and a perfusion lung scan were performed, and both were within normal limits. An ophthalmology consultation was obtained, at which time a gas bubble was noted in the anterior chamber.

Ophthalmic examination on 18 October 1981 revealed an opaque cornea and a very tense globe. An anterior chamber paracentesis was performed and approximately $0.10 \mathrm{ml}$ of chocolate coloured fluid was obtained. This material was plated on blood and chocolate agars and was submitted for anaerobic culture.

On 19 October 1981 the patient died. A general necropsy, performed $21 / 2$ hours after death, revealed severe atherosclerotic cardiovascular disease, dissecting aortic aneurysm, severe brochopeneumonia, and changes consistent with sepsis. The aortic aneurysm was not ruptured and did not appear to be 
the cause of death. No recent myocardial infarction was found. Culture of the anterior chamber aspirate grew a pure colony of Clostridium septicum.

PATHOLOGICAL EXAMINATION OF THE EYE The globe was examined under the dissecting microscope in the pathology laboratory. No penetrating injury could be identified. The corneal surface was opaque, extremely irregular, and thin in the centre. On transverse sectioning the globe was observed to be diffusely necrotic. A small amount of purulent, coffee coloured fluid was found in the anterior chamber and vitreous cavity. The inner surface of the eye was pale and extensively necrotic. The retina was not identified. Instead the inner surface of the globe was irregular, elevated, and bullous in appearance.

Histological examination of the eye showed that all ocular tissues were involved in an acute necrotising inflammatory process (Fig. 1). The cornea and sclera (Fig. 2) were diffusely infiltrated with acute inflammatory cells and had undergone severe collagen degeneration and necrosis. Scleral and episcleral blood vessels were surrounded by acute inflammation and their walls were partially necrotic. The central cornea was extremely thin, with total absence of the epithelium and more than half of the stroma. The ciliary body and iris were extensively inflamed and necrotic. The choroid was detached in some segments and was also involved in the diffuse necrosis; in some areas only the framework of the vascula- ture was left intact. The choroidal blood vessels were found to contain many thromboemboli (Fig. 3). Only fragments of retinal structures showing severe necrosis could be identified in certain segments of the globe. In addition to its necrotic appearance the tissue contained empty spaces possibly secondary to gas-producing bacteria. The overall bullous appearance of the retina and choroid was also consistent with extensive oedema and gas production. A few clusters of Gram-positive bacteria were identified, but these did not have the spindle, drumstick appearance of Clostridium septicum and did not contain spores.

\section{Discussion}

Clostridial infections of the eye are devastating. Since Leavelle's' extensive review of 53 previously reported cases as well as three of his own nine additional cases have been reviewed by Frantz et al. ${ }^{5}$ All previously reported cases of gas gangrene panophthalmitis followed pentrating injuries to the eye and the offending organism was Clostridium perfringens. Frantz and colleagues' report included a 68-year-old white male who presented with fever, pain in the right upper quadrant of the abdomen, and panophthalmitis of the left eye. Unlike the previously described cases their case resulted from endogenous infection of the eye complicating perforation of a gangrenous gallbladder.

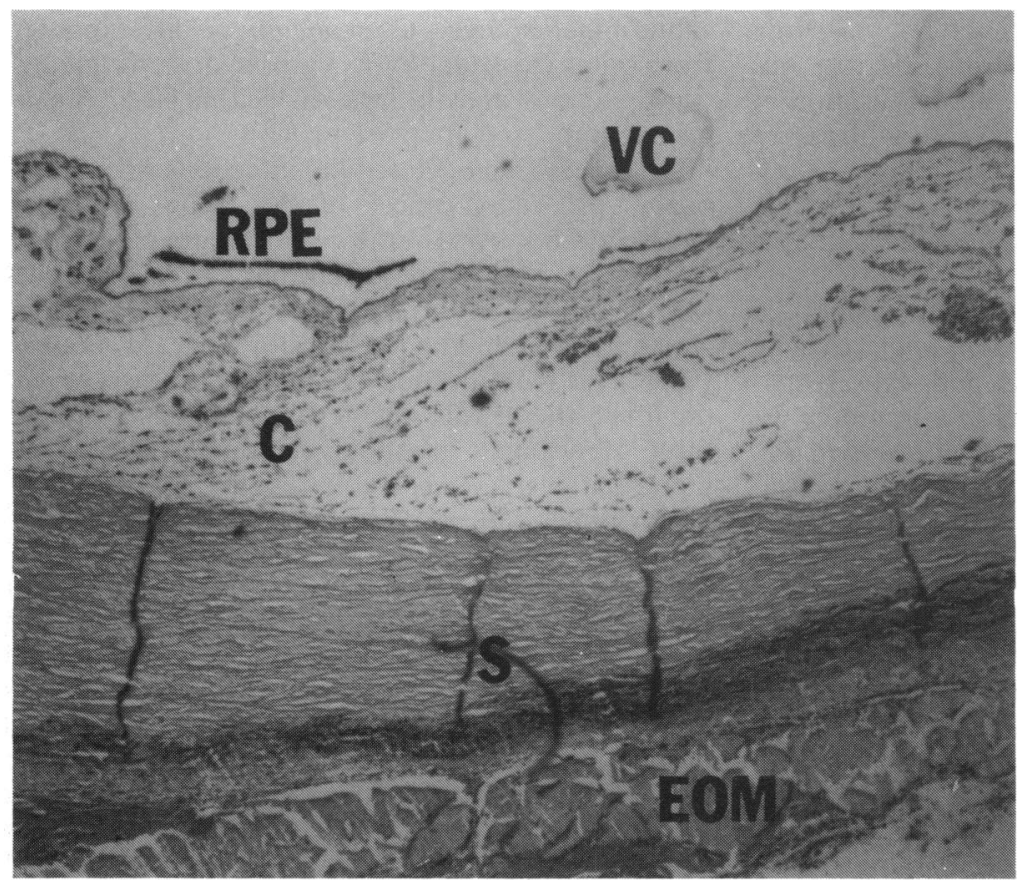

Fig. 1 Extensive oedema and necrosis of retina and choroid. Retina is totally necrotic. Only a short segment of detached retinal pigment epithelium (RPE) is seen in the figure. VC: vitreous chamber; C: choroid; S: sclera; EOM: extraocular muscle. (Haematoxylin and eosin, $\times 40$ ). 
Fig. 2 Diffuse infiltration of acute inflammatory cells and necrosis in retina $(\mathrm{R})$, choroid $(\mathrm{C})$, and sclera (S). $(H$ and $E, \times 100)$.

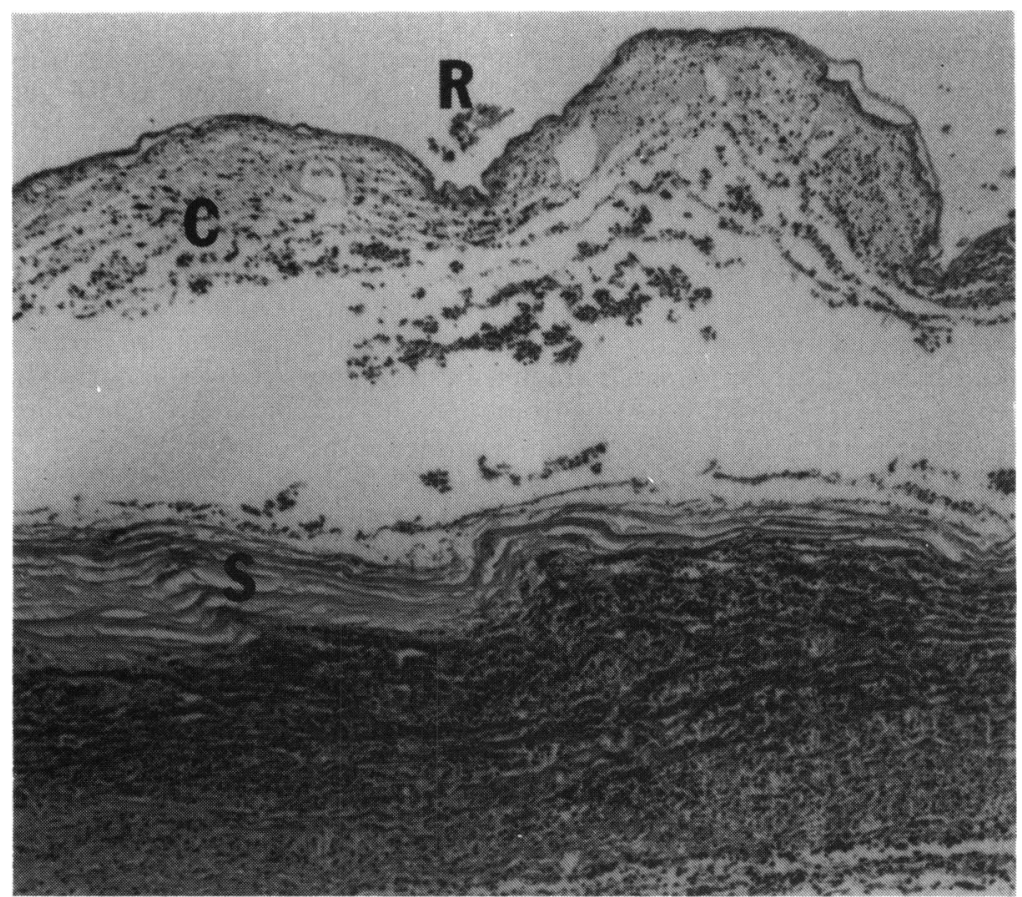

The clinical picture of clostridial panophthalmitis described by Leavelle is one of severe pain and rapid loss of vision within 12 hours of injury. An explosive panophthalmitis with chemosis and swelling of the lid is usually apparent by 18 hours. Hypopyon, ring abscess of the cornea, coffee coloured discharge, gas bubbles in the anterior chamber, immobilisation of the globe, and a total loss of fundus reflex with loss of vision were signs usually evident by 24 hours. Conventional antibiotic therapy has been entirely impotent against this disastrous disease, even though some cases were seen very early.

For practical purposes the clostridia may be divided into three groups: $C$. botulinum causing botulism, $C$. tetani causing tetanus, and several types of clostridia causing gas gangrene, commonly $C$. perfringens, $C$. novyi, C. septicum, $C$. histolyticum, and $C$. fallax. All the clostridia are large Grampositive rod-like anaerobic bacteria with a larger spore-forming end, giving them a drumstick appearance. The clostridia which may be considered as potential causes of gas gangrene panophthalmitis produce powerful exotoxins. The most common member of this group of organisms is $C$. perfringens (C. welchii); next are $C$. novyi and $C$. septicum in degree of frequency of isolation from war wounds.

C. septicum, an obligate anaerobe, is B-haemolytic on blood agar and highly motile. Spreading growth can be overlooked, as isolates may form only a thin film on the culture plate without the definition of distinct colonies. Smears from cultures show Grampositive sporulating rods with oval terminal and subterminal spores. C. septicum is differentiated from other clostridia by its carbohydrate fermentation reactions and its lack of lecithinase or lipase production.

An association of clostridial infections with malignancy, especially cancer of the colon, has been demonstrated by several case reports. ${ }^{n-4}$ In 1969 Alpern and Dowell ${ }^{\mathrm{t}}$ reported that 23 of 27 patients $(85 \%)$ with $C$. septicum infections whose cultures were referred to the Center for Disease Control (CDC) for identification had malignant disease. Of the four patients who did not have malignant disease three developed intestinal tumours one to two months after the $C$. septicum was cultured.

Koransky et al. ${ }^{7}$ reported on the clinical significance of $C$. septicum bacteraemia. The medical records of 59 patients were reviewed; $42(71 \%)$ of these patients had malignant disease. Half had haematological malignant disease and one half had solid tumours. Of the 21 patients with solid tumours $14(67 \%)$ had cancer of the colon. The caecum and distal ilieum were thought to be the most probable portals of entry for $C$. septicum bacteraemia among the 28 patients examined at necropsies. They reported one patient with distant metastatic infection 


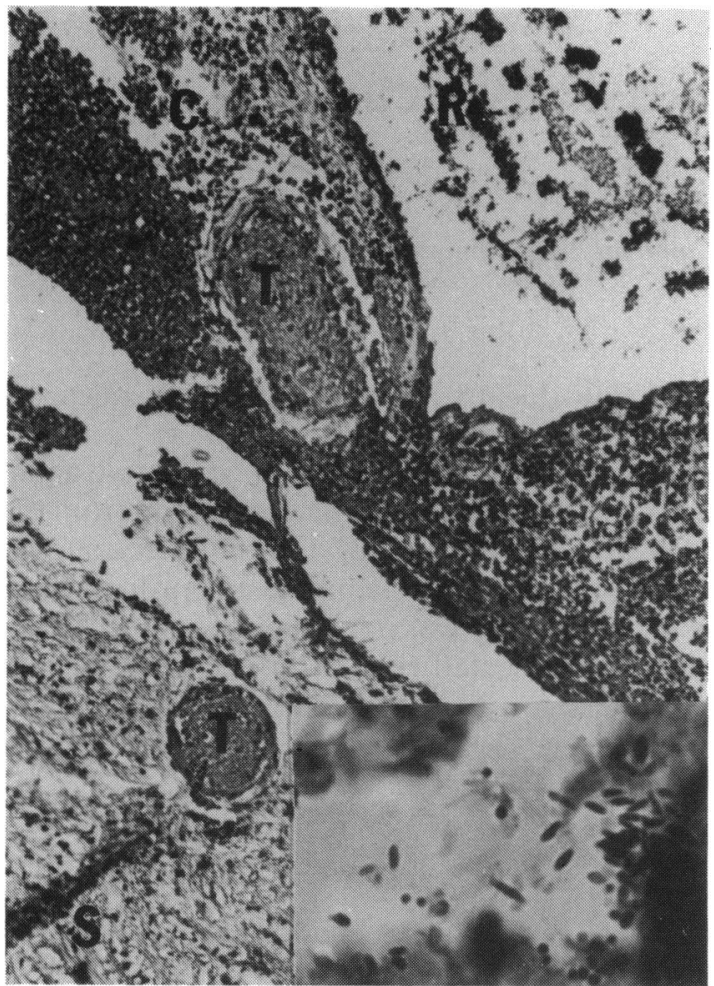

Fig. 3 Oedema, necrosis, and diffuse acute inflammatory cell infiltration of the retina, choroid, and sclera with multiple thromboemboli $(\mathrm{T})$ in choroidal and scleral blood vessels. $\mathrm{R}$ : necrotic retina; C: choroid; S: sclera. ( $H$ and $E, \times 85)$. Insert: Organisms, identified in culture as Clostridium septicum, within necrotic areas of the sclera. (Gram stain, $\times 850$, oil immersion).

of the eye who had an enucleation and then was later found to have adenocarcinoma of the colon.

Further results of their study showed that of the 17 patients with no detectable malignancy six had severe atherosclerotic cardiovascular disease with a gangrenous extremity or gangrenous bowel. Infection with $C$. septicum was the apparent cause of death in 40 patients $(68 \%)$. All surviving patients were given penicillin, cephalothin, chloramphenicol, or carbenicillin (antibiotics to which clostridia have been found to be sensitive ${ }^{\text {"II) }}$ soon after they were admitted to the hospital.

Post-mortem examination of our patient, who apparently developed spontaneous gas gangrene panophthalmitis due to Clostridium septicum, failed to disclose an occult malignant tumour, which must be ruled out when $C$. septicum is cultured from a 'metastatic' infection outside the bowel. ${ }^{\circ}$ However, she did have severe atherosclerotic cardiovascular disease, as did six of 17 patients without malignant disease in Koransky and colleague's series.' It has been suggested that arteriosclerosis may have a role in the development of ischaemia and tissue necrosis, which are important predisposing factors for clostridial growth and gangrene formation.?

Although a positive (ante-mortem) blood culture was not obtained in our case, we attribute this to antimicrobial therapy instituted prior to the sampling. Fever, especially when accompanied by gas gangrene, tissue necrosis, and/or the signs of shock which characterised our patient's hospital course, should suggest possible septicaemia caused by toxic bacteria such as clostridium.

The importance of early recognition and prompt therapy in patients with Clostridium septicum infection cannot be overemphasised. When these patients are admitted to hospital they are acutely ill and toxic. Their clinical course is usually fast downhill, and unless the appropriate antibiotics are administered soon after admission the outcome is fatal. Blood cultures, both aerobic and anaerobic, should be performed rapidly, and therapy should be instituted before the results of the cultures are received. Penicillin remains the drug of choice, but some patients may be treated successfully with chloramphenicol, carbenicillin, or cephalothin.

\section{References}

1 Lcavelle RB. Gas gangrenc panophthalmitis. Arch Ophthalmol 1955; 53: 634-42.

2 Walsh TJ. Clostridial ocular infections. Case report of gas gangrene panophthalmitis. Br J Ophthalmol 1965; 49: 472-7.

3 Kurz GH, Weiss JF. Gas gangrene panophthalmitis. $B r J$ Ophthalmol 1969; 53: 323-6.

4 Levitt JM, Stam J. Clostridium perfringens panophthalmitis. Arch Ophthalmol 1970; 84: 227-88.

5 Frantz JF, Lemp MA, Font RL, Stonc R, Eisner E. Acutc endogenous panophthalmitis causcd by Clostridium perfringens. Am J Ophthalmol 1974; 78: 295-303.

6 Alpern RJ, Dowell VR. Clostridium septicum infections and malignancy. JAMA 1969; 209: 385-8.

7 Koransky JR, Stargel MD, Dowell VR. Clostridium septicum bacteremia. Am J Med 1979; 66: 63-6.

8 Katlic MR, Derkac WM, Colcman WS. Clostridium septicum infection and malignancy. Ann Surg 1981; 193: 361-4.

9 Schaaf RE, Jacobs N, Kclvin FM, et al. Clostridium septicum infection associated with colonic carcinoma and hematologic abnormality. Radiology 1980; 137: 625-7.

10 Gabay EL, Rolfe RD, Fincgold SM. Susceptibility of Clostridium septicum to 23 antimicrobial agents. Antimicrob Agents Chemother 1981; 20: 852-3. 